\title{
Benefício de aposentadoria por invalidez previdenciária em portadores de doença renal crônica submetidos a transplante renal
}

\author{
Social Security disability benefit eligibility after kidney transplantation
}

\author{
Talita Zerbini' ${ }^{1}$ Magda Miranda Pires Araki
}

DOI: http://dx.doi.org/10.11606/issn.2317-2770.v19i2p86-9

Zerbini TZ, Araki MMP. Benefício de aposentadoria por invalidez previdenciária em portadores de doença renal crônica submetidos a transplante renal. Saúde, Ética \& Justiça. 2014;19(2):86-9.

RESUMO: A doença renal crônica é uma síndrome clínica causada pela perda progressiva e irreversível das funções renais. No Brasil, a Previdência Social utiliza a taxa de filtração glomerular da creatinina como um dos critérios para estabelecer o grau de capacidade laboral do segurado. Entretanto, com o transplante renal, há a possibilidade de melhora da qualidade de vida do segurado, existindo a possibilidade de reintegrá-lo no meio socioeconômico. Após o período inicial de transplante, o segurado pode estar capaz para retornar ao trabalho. Sendo assim, o presente trabalho aborda os aspectos que devem ser pesquisados pelo médico perito em uma perícia previdenciária oficial de um segurado submetido a transplante renal, tais como taxa de filtração e avaliação das consequências da terapia imunossupressora instituída.

DESCRITORES: Insuficiência Renal Crônica; Seguro por Invalidez; Qualidade de Vida; Pensões.

\footnotetext{
1. Santa Casa, Fundação ABC, IML

2. Médica com especialização em Medicina Legal pelo Hospital das Clínicas da Faculdade de Medicina da Universidade de São Paulo.

Endereço para correspondência: Talita Zerbini. Avenida Doutor Arnaldo, 455, Cerqueira César - São Paulo/SP. CEP 01246-903. E-mail: tazerbini@yahoo.com.br
} 


\section{INTRODUÇÃO}

Insuficiência Renal Crônica (IRC) é
uma síndrome metabólica decorrente
de perda progressiva, geralmente lenta, da capacidade excretória renal. A função de excreção de catabólitos é resultante principalmente da filtração glomerular, sendo a IRC resultante da redução da taxa de filtração glomerular (TFG), que pode ser avaliada clinicamente pela medida do "clearance" de creatinina em exame de urina 24 horas $^{1}$. Em pacientes com IRC, a filtração pode chegar a menos de $15 \mathrm{~mL} / \mathrm{min} / 1,73 \mathrm{~m}^{2}$, situação em que o tratamento dialítico ou o transplante renal se fazem necessários. $\mathrm{O}$ termo doença renal crônica (DRC) é utilizado para denominação das doenças renais que causam tal insuficiência ${ }^{1}$.

Para efeitos clínicos, epidemiológicos, didáticos e conceituais, a DRC é dividida em seis estágios funcionais, de acordo com o grau de função renal do paciente, quais sejam ${ }^{2}$ :

- Estágio 0 - Fase de função renal normal sem lesão: contempla indivíduos que fazem parte dos grupos de risco para o desenvolvimento da insuficiência renal: diabéticos, hipertensos, indivíduos com histórico familiar de DRC, entre outros (TFG $=90 \mathrm{~mL} / \mathrm{min} / 1,73 \mathrm{~m}^{2}$ ou mais);

- Estágio 1 - Fase de lesão com função renal normal: trata-se de indivíduos que possuem lesão renal em estágio inicial, mas mantém níveis seguros de filtração glomerular (TFG entre 60 e $89 \mathrm{~mL} / \mathrm{min} / 1,73 \mathrm{~m}^{2}$ );

- Estágio 2 - Fase de insuficiência renal funcional ou leve: Nesta fase, os rins ainda são capazes de manter o controle dos fluidos corporais. No entanto, já há perda da função renal, a qual é detectada apenas por meio de métodos eficientes de avaliação funcional (TFG entre 45 e $59 \mathrm{~mL} / \mathrm{min} / 1,73 \mathrm{~m}^{2}$ );

- Estágio 3 - Fase de insuficiência renal laboratorial ou moderada: o estado clínico do paciente é considerado bom, mas observam-se, por meio de avaliação laboratorial simples, alterações nos níveis de creatinina plasmáticos e de ureia (TFG entre 30 e $44 \mathrm{~L} / \mathrm{min} / 1,73 \mathrm{~m}^{2}$ );

- Estágio 4 - Fase de insuficiência renal clínica ou severa: paciente apresenta sinais e sintomas marcados de uremia, tais como sintomas digestivos, fraqueza, malestar, anemia, edema e hipertensão arterial (TFG entre 15 e $29 \mathrm{~mL} / \mathrm{min} / 1,73 \mathrm{~m}^{2}$ );

- Estágio 5 - Fase terminal de insuficiência renal crônica: o rim torna-se incapaz de regular o meio interno e configura-se perda significativa da função renal, incompatível com a vida (TFG menor do que $15 \mathrm{~L} /$ $\left.\min / 1,73 \mathrm{~m}^{2}\right)$.

A doença renal crônica traz consigo uma série de questões que marcam a vida do indivíduo a partir do diagnóstico, sendo comuns as manifestações psíquicas que acarretam alterações na interação social, não somente do paciente como também da família que o acompanha ${ }^{3}$.
São várias as causas de IRC, sendo as mais comuns a glomerulonefrite crônica, nefropatia túbulo-intersticial crônica (pielonefrite), necrose cortical renal, hipertensão arterial grave, processos renais obstrutivos crônicos, diabetes, amiloidose, lúpus eritematoso disseminado e doenças hereditárias tais como rins policísticos e síndrome de Alport ${ }^{1}$.

Os tratamentos disponíveis para o manejo da doença renal crônica terminal não são curativos, mas substitutivos da função renal perdida, aliviando os sintomas da doença ${ }^{4}$.

O transplante renal é uma importante opção terapêutica para o paciente com insuficiência renal crônica, tanto do ponto de vista médico quanto social ou econômico. Está indicado quando houver insuficiência renal crônica em fase terminal, estando o paciente em diálise ou mesmo em fase pré-dialítica (pré-emptivo), considerando-se clearance de creatinina $<20 \mathrm{ml} / \mathrm{min} / 1,73 \mathrm{~m}^{2}$ de superfície corporal ${ }^{5}$. Como alternativa de tratamento, o transplante renal tem mostrado, a longo prazo, benefícios à saúde e exibe atrativos no aspecto custo $\mathrm{x}$ benefício. $\mathrm{O}$ transplante renal como intervenção terapêutica é o que fornece maior possibilidade de independência ${ }^{6,7}$.

De acordo com o anuário estatístico disponibilizado pelo Instituto Nacional do Seguro Social (INSS), foram concedidos ao todo 172.609 benefícios de aposentadoria por invalidez no ano de 2012, sendo 2.905 (1,7\% do total) em decorrência de transtornos do aparelho geniturinário. Segundo o disposto no parágrafo único do artigo 46 do Decreto 3.048/99, o aposentado por invalidez pelo INSS poderá ser convocado a qualquer tempo para verificação da manutenção de sua invalidez, sendo obrigado a se submeter aos exames médico-periciais a cada dois anos ${ }^{8}$.

Desse modo, o presente trabalho tem por objetivo rever os critérios clínicos que devem ser avaliados em perícia médica previdenciária de segurados que foram submetidos a transplante renal.

\section{MÉTODO}

O trabalho foi realizado por meio de revisão bibliográfica em base de dados Lilacs/Scielo e Pub Med utilizando-se os descritores segundo o $\mathrm{DeCs}^{\circledR}$ : "insuficiência renal crônica", "transplante renal", "seguro por invalidez", "qualidade de vida" e "benefícios de aposentadoria", com seus respectivos descritores em inglês: "renal insufficiency, chronic", "kidney transplantation", "insurance, disability", "quality of life" e "pensions". Os artigos analisados foram publicados entre os anos de 2002 e 2012. Além disso, foram analisadas a lei federal 8213/91 e alterações e o Decreto n. 3.048/99, que dispõem sobre a previdência social no Brasil.

\section{RESULTADOS E DISCUSSÃO}

O médico perito deverá, ao registrar o diagnóstico, 
identificar o tipo de nefropatia, em seguida, a indicação da presença ou não de nefropatia grave e avaliar o grau de capacidade laboral, lembrando que a ausência de incapacidade não implica ausência de doença ou lesão, devendo ser considerada a repercussão da doença ou lesão no desempenho das atividades laborais ${ }^{9}$. De acordo com o previsto em lei, o benefício de aposentadoria por invalidez é concedido àqueles que apresentam incapacidade laborativa classificada como total e permanente ${ }^{7}$.

$\mathrm{O}$ transplante renal é feito com rins de doadores vivos ou doadores cadáveres. $\mathrm{O}$ transplante com doador vivo pode ser feito com indivíduos consanguíneos, familiares até o quarto grau (grau I - pais e filhos; grau II - avós e irmãos; grau III - tios e sobrinhos; grau IV - primos e filhos de tios consanguíneos) e também de cônjuges. O transplante com órgãos de doadores vivos não relacionados ou amigos só é permitido depois de extenso trâmite, que envolve justificativa médica, autorização ética e judicial para o procedimento ${ }^{5}$.

O transplante com doador vivo é realizado em caráter eletivo com o doador disponível ou preferencialmente com o de melhor compatibilidade com relação aos antígenos do complexo antígeno leucocitário humano (HLA). A definição e abordagem criteriosas na seleção do doador vivo são fundamentais para a segurança do doador ${ }^{10}$.

Transplantes com doadores cadáveres são efetuados em pacientes urêmicos terminais em tratamento dialítico inscritos na lista única estadual ${ }^{11}$. Nos últimos anos, ocorreu grande avanço no arsenal de drogas destinadas à prevenção e ao tratamento das rejeições dos órgãos transplantados. As drogas imunossupressoras tornaramse progressivamente mais potentes e seletivas. Diversos agentes imunossupressores estão disponíveis atualmente, e esta disponibilidade permite que se individualize a terapia imunossupressora, fornecendo aos pacientes o regime mais adequado considerando principalmente o tipo e a compatibilidade do doador, o risco de rejeição, a idade, a raça e a presença de comorbidades ${ }^{5}$. Quanto à morbidade, mortalidade e qualidade de vida, o transplante renal constitui-se na melhor alternativa para o tratamento de doença renal crônica ${ }^{11}$.

Segundo o Manual de Perícia Oficial em Saúde do Servidor Público Federal (2010), as nefropatias que cursam com insuficiência renal leve, estágios 1 e 2, não são enquadradas como nefropatias graves para fins periciais. Já as nefropatias que cursam com insuficiência renal moderada, estágio 3, são enquadradas como nefropatias graves quando acompanhadas de sintomas e sinais que determinam a incapacidade laborativa do periciado, tais como edema importante, dispneia, enjoo constante e fraqueza. As nefropatias classificadas como estágios 4 e 5 são enquadradas como nefropatias graves ${ }^{9}$.

O tempo de transplante parece ser uma variável importante na qualidade de vida dos transplantados. Os primeiros seis meses após a cirurgia são preocupantes, pelo risco mais alto de rejeição neste período, visitas constantes e necessárias ao hospital e ajustamento do paciente à medicação ${ }^{4}$. O tratamento imunossupressor é a principal razão para que o paciente transplantado permaneça sem trabalho remunerado ${ }^{12}$. Porém, a depender de sua função, o paciente renal crônico transplantado pode ser considerado capaz de retorno ao trabalho.

Segundo o Decreto n. 3.048/99, o beneficiário pode ser convocado para nova perícia médica e ter seu benefício suspenso, além de poder ser iniciado processo de habilitação e de reabilitação profissional a ser desenvolvido por meio das funções básicas de avaliação do potencial laborativo ${ }^{13}$.

\section{CONCLUSÕES}

Completado o período de seis meses após o transplante renal, o doente renal crônico poderá ser considerado capaz para o trabalho se mantiver a taxa de filtração glomerular acima de $45 \mathrm{~mL} / \mathrm{min} / 1,73 \mathrm{~m}^{2}$, devendo o médico perito verificar se a terapia imunossupressora instituída permite que o mesmo realize sua função habitual ou se deverá ser encaminhado ao processo de reabilitação profissional, cabendo ao médico do trabalho analisar a adequação de suas atividades laborais.

Zerbini TZ, Araki MMP. Social Security disability benefit eligibility after kidney transplantation.Saúde, Ética \& Justiça.

2014;19(2):86-9.

\begin{abstract}
Chronic kidney disease is a clinical syndrome in which there is progressive and irreversible loss of kidney function. In Brazil, the social security agency uses the glomerular filtration rate of creatinine as one of the criteria for establishing the degree of disability of the insurance claimant. However, with kidney transplantation it is possible to improve the quality of life of the claimant, making it possible for him/her to resume his/her social role. After the initial period of transplantation, the claimant may be capable of returning to work. Thus, this paper discusses the aspects that must be investigated by the expert physician, as an official social pension expert, regarding the conditions of an insurance claimant whom has had a kidney transplant.
\end{abstract}

KEY WORDS: Renal Insufficiency; Chronic; Insurance; Disability; Quality of Life; Pensions. 


\section{REFERÊNCIAS}

1. Draibe SA, Ajzen H. Guia de Medicina Ambulatorial e Hospitalar da Unifesp - EPM - Nefrologia. $3^{\text {a }}$ ed. São Paulo: Manole; 2011. p.1-8. p.327-42.

2. Júnior JER. Doença Renal Crônica: Definição, Epidemiologia e Classificação. J Bras Nefrol. 2004;26(3):13

3. Higa K, Kost MT, Soares DM, Morais MC, Polins BRG Qualidade de vida de pacientes portadores de insuficiência renal crônica em tratamento de hemodiálise. Acta Paul Enferm. 2008;21(número especial):203-6. DOI: http:// dx.doi.org/10.1590/S0103-21002008000500012

4. Ravagnani LMB, Domingos NAM, Miyazaki MCOS. Qualidade de vida e estratégias de enfretamento em pacientes submetidos a transplante renal. Estudos de Psicologia. 2007;12(2):177-84

5. Brasil. Presidência da República. Ministério da Saúde Portaria $\mathrm{n}^{\mathrm{0}}$ 666, de 17 de julho de 2012. Aprova o Protocolo Clínico e Diretrizes Terapêuticas - Imunossupressão no Transplante Renal. Diário Oficial da União, Brasília (DF). 201220 jul.;Seção 1:69.

6. Lôbo MCSG, Bello VAO. Reabilitação Profissional pós Transplante Renal. J Bras Nefrol. 2007;29(1):29-32.

7. Bastos Mg, Kirstjan, GM. Doença renal crônica: importância do diagnóstico precoce, encaminhamento imediato e abordagem interdisciplinar estruturada para melhora do desfecho em pacientes ainda não submetidos à diálise. J Bras Nefrol. 2011;33(1):93-108. DOI: http:// dx.doi.org/10.1590/S0101-28002011000100013

8. Brasil. Presidência da República. Ministério da Previdência Social. Lei no 8.213 de 24 de julho de 1991. Dispõe sobre Planos de Benefícios da Previdência Social e dá outras providências. Diário Oficial da União, Brasília (DF). 1991 25 jul. p. 14809.

9. Brasil. Presidência da República. Ministério do Planejamento, Orçamento e Gestão. Manual de perícia oficial em saúde do servidor público federal. Brasília (DF): Ministério do Planejamento, Orçamento e Gestão; 2010.

10. Pereira LC, Chang J, Fadil-Romão MA, Abensur H, Araújo MRT, Noronha IL, et al. Qualidade de vida relacionada à saúde em paciente transplantado renal. J Bras Nefrol. 2003;25(1):10-6.

11. Manfro RC, Carvalhal GF. Transplante renal. Rev AMRIGS. 2003;47(1):14-9.

12. Bommer J. Prevalence and socio-economic aspects of chronic kidney disease. Nephrol Dial Transplant. 2002;17(Suppl 11):8-12. DOI: 10.1093/ndt/17.suppl_11.8

13. Brasil. Decreto $\mathrm{n}^{0} 3.048$, de 06 de maio de 1999. Aprova o Regulamento da Previdência Social, e dá outras providências. Diário Oficial da União, Brasília (DF). 1999 25 mai. 